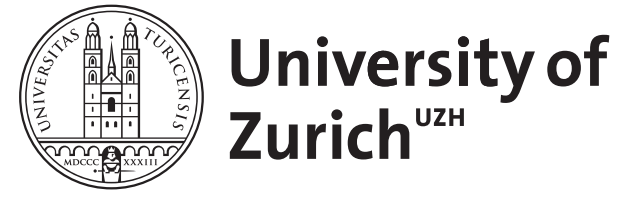
Archive

University of Zurich

University Library

Strickhofstrasse 39

CH-8057 Zurich

www.zora.uzh.ch

Year: 2012

\title{
Probiotika bei gastrointestinalen Erkrankungen
}

Braun, S ; Budak, K ; Vavricka, Stephan R

DOI: https://doi.org/10.1024/1661-8157/a001041

Posted at the Zurich Open Repository and Archive, University of Zurich

ZORA URL: https://doi.org/10.5167/uzh-67484

Journal Article

Accepted Version

Originally published at:

Braun, S; Budak, K; Vavricka, Stephan R (2012). Probiotika bei gastrointestinalen Erkrankungen. Praxis, 101(17):1077-1085.

DOI: https://doi.org/10.1024/1661-8157/a001041 


\title{
Probiotika bei gastrointestinalen Erkrankungen
}

\author{
Hintergrund und Definition ((Ü1))
}

Der Intestinaltrakt ist mit einer Vielzahl von Bakterien besiedelt, die die Darmflora bilden und die normale Darmfunktion unterstützen. Ist das natürliche Gleichgewicht zwischen apathogenen und potenziell pathogenen Erregern gestört, kann die Entstehung gastrointestinaler Erkrankungen begünstigt werden. Die gezielte Manipulation der Darmflora zu therapeutischen Zwecken ist Gegenstand intensiver Forschung und aufgrund der Vielzahl an Einsatzmöglichkeiten auch im ärztlichen Alltag von Interesse. Probiotika sind definiert als lebende, nicht pathogene Mikroorganismen, die die endogene intestinale Flora modifizieren und einen positiven Effekt auf den Wirt haben. Sie werden für eine Vielzahl von Indikationen eingesetzt, obwohl die Datenlage sehr heterogen ist. Im Folgenden soll ein Überblick gegeben werden, bei welchen gastrointestinalen Erkrankungen bestimmte Probiotika sinnvoll eingesetzt werden können.

Im Artikel verwendete Abkürzungen

$\begin{array}{ll}\text { 5-ASA } & \text { 5-Aminosalicylsäure (Mesalazin) } \\ \text { AAD } & \text { Antibiotika-assoziierte Diarrhö } \\ \text { CDAD } & \text { Clostridium-difficile-assoziierte Diarrhö } \\ \text { CFU } & \text { Kolonie-bildende Einheiten } \\ \text { E. coli } & \text { Escherichia coli } \\ \text { LGG } & \text { Lactobacillus rhamnosus GG (Gorbach-Goldin) } \\ \text { S. boulardii } & \text { Saccharomyces boulardii }\end{array}$

Übersicht über Arten und Wirkung von Probiotika ((Ü1))

Probiotika beeinflussen über verschiedene Mechanismen die intestinale Flora (Tab. 1): Durch die temporäre Besiedelung des Darms durch Probiotika kommt es zu einem kompetitiven Wettbewerb und einer grösseren bakteriellen Diversität, was das Wachstum pathogener Keime erschwert. Häufig zeigen sie eine antimikrobielle Aktivität durch eine säurebedingte Senkung des intestinalen $\mathrm{pH}$-Wertes oder durch Produktion antibakterieller Toxine und Enzyme. Die intestinale Barriere wird aufrechterhalten, indem die Anhaftung pathogener Bakterien an die Darmwand erschwert und die Durchlässigkeit für Toxine und Keime reduziert wird. Bei einigen Probiotika besteht eine positive immunmodulatorische Wirkung, beispielsweise durch Induktion protektiver Zytokine (wie IL10, TGF-beta), Hemmung proinflammatorischer Zytokine (wie TNF- $\alpha$ ), vermehrte Sekretion von IgA und vermehrte Phagozytose-Aktivität. In der Schweiz sind Probiotika sowohl als Nahrungsergänzungsmittel in Lebensmitteln als auch als spezielle Präparate zur Behandlung und Prophylaxe bestimmter Erkrankungen erhältlich (Tab. 2).

Tab. 1: Wirkungsmechanismen von Probiotika

Verbesserung der intestinalen Barriere durch 
- $\quad$ verminderte Anhaftung pathogener Bakterien an die Darmwand

- $\quad$ verminderte Durchlässigkeit für Toxine und Keime

Beeinflussung des Immunsystems durch

- Induktion protektiver Zytokine (z.B. IL-10, TGF-beta)

- $\quad$ Suppression proinflammatorischer Zytokine (z.B. TNF-a)

- vermehrte Sekretion von $\lg \mathrm{A}$

- $\quad$ vermehrte Phagozytose-Aktivität

\section{Antimikrobielle Wirkung durch}

- grössere Diversität der Darmflora

- Senkung des pH-Werts durch Säureproduktion

- Produktion antibakterieller Enzyme und Toxine

Tab. 2: Probiotika

Probiotika in Joghurts und anderen Milchprodukten

\begin{tabular}{|c|c|}
\hline Lactobazillen & \\
\hline Aktifit $^{(\Theta)}($ Emmi) & $\begin{array}{l}\text { Lactobacillus rhamnosus Gorbach-Goldin (LGG), } \\
\text { Lactobacillus reuteri, Lactobacillus plantarum }\end{array}$ \\
\hline Actimel $^{\circledR}$ (Danone) & $\begin{array}{l}\text { Lactobacillus casei defensis, Lactobacillus acidophilus, } \\
\text { Lactobacillus paracasei }\end{array}$ \\
\hline $\mathrm{LC}^{\circledR}{ }^{\circledR}$ (Nestlé) & Lactobacillus johnsonii \\
\hline Probiotika in Joghurt-Starterkulturen & $\begin{array}{l}\text { Lactobacillus bulgaricus } \\
\text { Streptococcus thermophilus }\end{array}$ \\
\hline Klinisch geprüfte probiotische, in der Schweiz erhä & che Keime \\
\hline Mutaflor $^{(B)}$ (Vifor) & Escherichia coli Nissle 1917 \\
\hline Bioflorin $^{(\otimes)} /$ Bioflorina $^{(}$(Sanofi-Aventis) & Enterococcus faecium SF68 \\
\hline Perenterol $^{(\Theta)}$ (Zambon), Sanaflor ${ }^{(} /$Florosan $^{(\Theta)}$ (Sandoz) & Saccharomyces boulardii \\
\hline $\begin{array}{l}\text { VSL\#3 }^{(B)} \text { (Sigma-Tau Pharmaceuticals), Gemisch aus } 9 \\
\text { Probiotika: }\end{array}$ & $\begin{array}{l}\text { Lactobacillus acidophilus, Lactobacillus plantarum } \\
\text { 299v, Lactobacillus paracasei, Lactobacillus } \\
\text { bulgaricus; Bifidobacterium breve, Bifidobacterium } \\
\text { longum, Bifidobacterium infantis, Streptococcus } \\
\text { thermophilus }\end{array}$ \\
\hline
\end{tabular}

Anwendung von Probiotika bei gastrointestinalen Erkrankungen ((Ü1))

\section{Probiotika bei Durchfallerkrankungen ((Ü2))}

Viele kleinere Studien und mehrere Metaanalysen deuten darauf hin, dass die Antibiotika-assoziierte Diarrhö (AAD) unter Einnahme von Probiotika seltener auftritt. Für eine generelle Empfehlung fehlen aber grössere Studien. In den letzten Metaanalysen reduzierte die Einnahme von Probiotika unter laufender antibiotischer Therapie die Inzidenz einer AAD um bis zu 52\%. Ein signifikanter Unterschied in der protektiven Wirkung 
zwischen den untersuchten Organismen, insbesondere zwischen Saccharomyces boulardii, Lactobacillus rhamnosus GG, Lactobacillus acidophilus und Lactobacillus bulgaricus, fand sich nicht [1,2]. Es existiert allerdings aufgrund der Heterogenität der verschiedenen untersuchten Studien kaum Information über optimale Dosis und optimalen Zeitpunkt der Probiotika-Einnahme.

Der Einsatz von Probiotika bei infektiöser Diarrhö bei Kindern wie bei Erwachsenen scheint trotz eingeschränkter Datenlage und nur mässigem Benefit sinnvoll [3]. Evidenz für einen Nutzen fand sich vor allem bei Kindern mit akutem Durchfall. So liess sich in mehreren systematischen Reviews eine Reduktion der Durchfall-Dauer nachweisen. Bei Kindern war vor allem Lactobacillus GG vorteilhaft [4]. Einige Probiotika (u.a. die Hefe Saccharomyces boulardii) schienen bei der Prophylaxe der Reisediarrhö eine leichte Risikoreduktion zu bewirken, andere (u.a. VSL\#3 ${ }^{\circledR}, L G G$ ) induzierten bei Kindern mit einer akuten Rotavirus-Infektion eine Besserung von Stuhlfrequenz und Hospitalisationsrate. Da infektiöse Durchfallerkrankungen meist selbstlimitierend sind und in Industrieländern bei sonst gesunden Personen selten zu Langzeitkomplikationen führen, Studien bei malnutrierten Durchfallerkrankten aus Entwicklungsländern dagegen weitgehend fehlen, bleibt der effektive Nutzen von Probiotika unklar. Genaue Informationen über Art, Dosis und Anwendungsdauer eines Probiotikums fehlen zudem. Bei Kindern schien eine Minimaldosis von 10 Billionen Kolonie-bildende Einheiten (CFU), innerhalb der ersten 48 Stunden verabreicht, v.a. bei Lactobacillen, wirksam zu sein [5].

Einen Sonderfall stellt die Clostridium-difficile-assoziierte Diarrhö (CDAD) dar. Nur wenige Studien untersuchten den Nutzen von Probiotika speziell bei Clostridium difficile. Angesichts der momentanen Datenlage können deshalb Probiotika bei den meisten Patienten für die Behandlung und Prophylaxe der CDAD nicht empfohlen werden. Ein systematischer Review von 2008 zeigte keinen Benefit von Probiotika zur Behandlung einer CDAD. Bei Patienten ohne signifikante Komorbiditäten kann allenfalls bei einem leichteren Verlauf eines CDAD-Rezidivs der Einsatz von Probiotika erwogen werden.

Bei älteren Patienten ohne signifikante Begleiterkrankungen, die unter antibiotischer Therapie standen, schien die zusätzliche Einnahme von Probiotika - geprüft wurden Lactobacillus acidophilus und Bifidobacterium bifidum - je nach Dosis zu einer niedrigeren Inzidenz einer CDAD zu führen und damit einen prophylaktischen Effekt zu haben. Ansonsten werden Probiotika für die Prävention einer CDAD nicht empfohlen [6,7].

\section{Probiotika bei chronisch-entzündlichen Darmerkrankungen ((Ü2))}

Der Nutzen von Probiotika bei chronisch-entzündlichen Darmerkrankungen ist eingeschränkt. Ihr Einsatz erfolgt im praktischen Alltag in enger Zusammenarbeit mit dem betreuenden Gastroenterologen. Beim Morbus Crohn können mangels Evidenz Probiotika weder für die Induktion noch für den Erhalt einer Remission empfohlen werden. Die Daten sind zu heterogen hinsichtlich Art und Dosis der verwendeten Probiotika, Studiendauer, Einschlusskriterien und gemessener Endpunkte, als dass Empfehlungen daraus abgeleitet werden könnten. In früheren Studien fanden sich mit Probiotika niedrigere Rezidivraten; untersucht wurden u.a. Escherichia coli Nissle 1917, Saccharomyces boulardii und VSL\# $3^{\circledR}[8,9]$.

Bei der Colitis ulcerosa liess sich beim Remissionserhalt und der Schubprophylaxe ein Effekt feststellen, bei der Remissionsinduktion dagegen nur eingeschränkt. E. coli Nissle 1917 zeigte sich bei der Schubprävention gleich effektiv wie 5-Aminosalicylsäure (Mesalazin, 5-ASA) und kann bei Unverträglichkeit oder ungenügendem Ansprechen von 5-ASA als Alternative erwogen werden [10]. Lactobacillus GG schien effektiver als die Standardtherapie mit Mesalazin (5-ASA) die Zeit bis zum nächsten Krankheitsschub zu verlängern ohne Senkung der Rezidivrate [11]. Die zusätzliche Einnahme von VSL\# $3^{\circledR}$ zeigte sich effektiver beim Remissionserhalt als die Standardtherapie alleine. Das Präparat schien bei milder bis mässig aktiver Colitis ulcerosa auch einen Benefit 
hinsichtlich Remissionsinduktion und Verminderung der Krankheitsaktivität zu bringen, allerdings ohne Besserung im histologischen Befund [12-14].

Bei Patienten mit ileoanaler Pouchanastomose nach totaler Kolektomie aufgrund Colitis ulcerosa oder familiärer adenomatöser Polyposis coli entwickelt sich häufig eine Pouchitis. Probiotika, im Speziellen VSL\# ${ }^{\circledR}$, scheinen bei der primären und sekundären Prävention einer solchen Pouchitis einen Nutzen zu bringen und sind damit eine Option ergänzend zur medikamentösen Standardtherapie [15].

Bei der Strahlenenteritis konnte in einer Metaanalyse kein Benefit festgestellt werden [16].

\section{Probiotika bei anderen gastrointestinalen Erkrankungen ((Ü2))}

Über den Einsatz von Probiotika bei chronischer Obstipation - ohne gleichzeitiges Colon irritabile - stehen zu wenig Daten zur Verfügung. Stuhlfrequenz und -konsistenz werden möglicherweise verbessert [17].

Auch für das Colon irritabile kann mangels klarer Evidenz keine Empfehlung für die Einnahme von Probiotika abgegeben werden. Ein kleiner Effekt besteht möglicherweise bei Diarrhö-dominiertem Colon irritabile, wo VSL\# $3^{\circledR}$ das Auftreten von Meteorismus reduzieren konnte. Auch Bifidobacterium infantis 35624 schien einen positiven Effekt zu haben. Alle entsprechenden Studien haben allerdings eine zu kurze Dauer, die meisten haben zudem methodische Fehler [18].

Der Nutzen von Lactase-haltigen Probiotika bei Laktose-Intoleranz war in einem Review unterschiedlich ausgeprägt, so dass weitere Studien zu einzelnen, potenziell effektiven Probiotika folgen müssen [19].

Die Einnahme von Probiotika zusätzlich zur Helicobacter-pylori-Eradikationstherapie schien in einigen Studien die Verträglichkeit der Behandlung zu verbessern. Saccharomyces boulardii verbesserte in einer Metaanalyse aus dem Jahr 2010 sogar die Eradikationsrate, so dass das Probiotikum als Zusatz zur Eradikationstherapie optional erwogen werden kann [20].

Bei Pankreatitis zeigte die Einnahme von Probiotika keinen Benefit hinsichtlich infektiöser Komplikationen, vielmehr zeigte sich entgegen früherer, vielversprechender Resultate, eine erhöhte Mortalität [21]. Probiotika werden bei Pankreatitis daher nicht empfohlen.

Bei der hepatischen Enzephalopathie können Probiotika derzeit nicht generell empfohlen werden. In zwei neueren systematischen Reviews wurden mögliche positive Effekte beschrieben; aufgrund der eingeschränkten Qualität der untersuchten Studien besteht aber derzeit ungenügende Evidenz für eine generelle Empfehlung $[22,23]$.

\section{Zusammenfassung ((Ü1))}

Probiotika werden in den meisten Fällen gut vertragen und sind bei einigen Erkrankungen ein vielversprechender Therapieansatz. Einige Einschränkungen bei der Verwendung bestehen jedoch: So ist die Studienlage weiterhin unübersichtlich und hinsichtlich Grösse, Methodik und Dauer der Studien eingeschränkt, so dass entsprechend der heterogenen Studienlage die Kosten für die meisten Probiotika nicht von der Krankenkasse übernommen werden. Grundsätzlich muss beachtet werden, dass die verschiedenen Probiotika unterschiedlich auf die Darmflora wirken und sich auch in ihrer Wirksamkeit bei verschiedenen Erkrankungen unterscheiden, so dass kein genereller, Probiotika-spezifischer Effekt besteht. Bei nur wenigen Indikationen (Tab. 3) wie der antibiotikaassoziierten oder infektiösen Diarrhö und der Rezidivprophylaxe einer Pouchitis kann anhand der bisherigen Publikationen von einem evidenten Nutzen bestimmter Probiotika ausgegangen und ihre Verwendung empfohlen werden. Hierbei sollten nur die für eine Indikation untersuchten Probiotika-Linien in der beschriebenen Dosis eingesetzt werden. Trotz insgesamt guter Verträglichkeit wurden bei signifikanten Komorbiditäten vereinzelt Bakteriämien (v.a. bei Lactobacillus rhamnosus GG) oder Fungämien (Saccharomyces boulardii) beschrieben, so dass Probiotika hier nicht eingesetzt werden sollten. 
Umfangreichere Studien zu erfolgversprechenden einzelnen Probiotika sind noch nötig, um in Zukunft die Wahl des entsprechenden Präparats zu erleichtern und mehr Informationen über optimale Dosierung und Therapiebeginn und -dauer zu gewinnen.

Tab. 3: Indikationen für den Einsatz von Probiotika

\begin{tabular}{|c|c|c|}
\hline Erkrankung & In Studien untersuchte Probiotika & Benefit \\
\hline \multicolumn{3}{|l|}{ Durchfallerkrankungen } \\
\hline Akuter Durchfall - Behandlung (Kinder) & $\begin{array}{l}\text { Saccharomyces boulardii } \\
\text { Lactobacillus rhamnosus GG (LGG) } \\
\text { Lactobacillus reuteri SD2112 }\end{array}$ & gut \\
\hline Akuter Durchfall - Behandlung (Erwachsene) & $\begin{array}{l}\text { Saccharomyces boulardii } \\
\text { LGG } \\
\text { Enterococcus faecium LAB SF68 }\end{array}$ & gut \\
\hline Prävention von Durchfall & $\begin{array}{l}\text { Saccharomyces boulardii } \\
\text { LGG }\end{array}$ & mittel \\
\hline Prävention von Antibiotika-assoziiertem Durchfall & $\begin{array}{l}\text { Saccharomyces boulardii } \\
\text { LGG } \\
\text { Kombination von Lactobacillus casei DN114 G01, } \\
\text { Lactobacillus bulgaricus, Saccharomyces } \\
\text { thermophilus }\end{array}$ & gut \\
\hline $\begin{array}{l}\text { Clostridium-difficile-assoziierte Diarrhö } \\
\text { - Rezidivprophylaxe } \\
\text { - Prophylaxe }\end{array}$ & $\begin{array}{l}\text { Saccharomyces boulardii } \\
\text { LGG } \\
\text { Saccharomyces boulardii } \\
\text { LGG } \\
\text { Kombination von Lactobacillus acidophilus und } \\
\text { Bifidobacterium bifidum }\end{array}$ & $\begin{array}{l}\text { mässig } \\
\text { mässig }\end{array}$ \\
\hline \multicolumn{3}{|l|}{ Chronisch-entzündliche Darmerkrankungen } \\
\hline M. Crohn & $\begin{array}{l}\text { Escherichia coli Nissle } 1917 \\
\text { Saccharomyces boulardii } \\
\text { LGG }\end{array}$ & schlecht \\
\hline $\begin{array}{l}\text { Colitis ulcerosa } \\
\text { - Rezidivprophylaxe/Erhaltungstherapie }\end{array}$ & $\begin{array}{l}\text { Escherichia coli Nissle } 1917 \\
\text { VSL\#3 }\end{array}$ & gut \\
\hline $\begin{array}{l}\text { Colitis ulcerosa } \\
\text { - Akuter Schub }\end{array}$ & $\begin{array}{l}\text { Escherichia coli Nissle } 1917 \\
\text { VSL\#3 }\end{array}$ & mittel \\
\hline $\begin{array}{l}\text { Pouchitis } \\
\text { - Prävention und Rezidivprophylaxe } \\
\text { - Akute Pouchitis }\end{array}$ & $\begin{array}{l}\text { VSL\#3 } \\
\text { VSL\#3 }\end{array}$ & $\begin{array}{l}\text { gut } \\
\text { schlecht }\end{array}$ \\
\hline Andere gastrointestinale Erkrankungen & & \\
\hline Colon irritabile & $\begin{array}{l}\text { Bifidobacterium infantis B5624 } \\
\text { VSL\#3 } \\
\text { Bifidobacterium animalis }\end{array}$ & $\begin{array}{l}\text { mittel } \\
\text { mittel } \\
\text { schlecht }\end{array}$ \\
\hline
\end{tabular}




\begin{tabular}{|c|c|c|}
\hline & Lactobacillus plantarum 299v & schlecht \\
\hline Akute Pankreatitis & Probiotika nicht empfohlen & schlecht \\
\hline Helicobacter pylori-Eradikationstherapie & Saccharomyces boulardii & gut-mittel \\
\hline Strahlen-Enteritis & $\begin{array}{l}\text { VSL\#3 } \\
\text { Lactobacillus acidophilus }\end{array}$ & $\begin{array}{l}\text { schlecht } \\
\text { schlecht }\end{array}$ \\
\hline \multicolumn{3}{|c|}{$\begin{array}{l}\text { Erklärung: Ähnlich zu Floch MH et al. sind bei gutem Benefit überzeugende positive Studien vorhanden, die eine } \\
\text { Empfehlung unterstützen. Bei «Benefit mittel» existieren positive kontrollierte Studien, aber auch einige negative S } \\
\text { die zu keinem Benefit kommen, bei «mässig» noch weniger positive Studien. Bei «Benefit schlecht» ist die Daten }\end{array}$} \\
\hline
\end{tabular}




\section{Fallbericht zu Probiotika bei gastrointestinalen Erkrankungen}

Anamnese des Patienten ((Ü1))

Wir berichten von einem 35-jährigen Mann mit seit drei Jahren diagnostizierter, distaler Colitis ulcerosa, den wir einen Monat nach dem letzten akuten Colitis-Schub in unserer hausärztlichen Sprechstunde sehen. Zum Remissionserhalt nahm er vor dem Schub 2x500 mg Salofalk $^{\circledR}$ (Mesalazin), worunter er über sechs Monate asymptomatisch war. Wegen vermehrter Tenesmen und schleimigem, teils blutigen Durchfall bis maximal fünf Mal täglich ohne Fieber suchte er vor einem Monat den betreuenden Gastroenterologen auf, wo sich der Patient afebril, normoton und normokard präsentierte, eine Druckdolenz im linken Unterbauch aufwies und erhöhte Entzündungszeichen (CRP $20 \mathrm{mg} / \mathrm{dl}$, BSR $32 \mathrm{~mm} / \mathrm{h}$, Leukozytenzahl $11,2 \times 10^{3} / \mathrm{ml}$, Thrombozyten $490 \times 10^{3} / \mathrm{ml}$ ) zeigte. Zur Akuttherapie wurde bei einem mässiggradigen Krankheitsschub die Tagesdosis auf $3 \mathrm{~g}$ Mesalazin erhöht. Hierunter waren die Symptome innerhalb von drei Wochen regredient, so dass die Dosis wieder reduziert werden konnte. Aktuell gibt er in der Sprechstunde keine Beschwerden mehr an. Allerdings habe er immer wieder nach Einnahme von Mesalazin Kopfschmerzen und Übelkeit bemerkt, so dass er gelegentlich die Tabletten nicht eingenommen habe, und fragt nach möglichen Alternativen. Im Internet habe er von Probiotika gelesen, die einen Effekt hätten.

\section{Weg zur Diagnose/Kommentar ((Ü1))}

Es bestand ein mittelgradiger Colitis-Schub mit entsprechender Anamnese (Tenesmen, blutige Diarrhö <6x/Tag), Klinik (u.a. lokale Druckdolenz, afebril) und Laborkonstellation (CRP, BSG, Leukozyten erhöht). Die Schubtherapie besteht bei distaler Colitis aus $1 \mathrm{~g}$ 5-ASA topisch, bei linksseitiger Beteiligung zusätzlich 3-4 g 5-ASA oral. 


\begin{tabular}{|c|c|}
\hline $\begin{array}{lc}\text { BSR } 16 \mathrm{~mm} / \mathrm{h} & (\text { Norm } \leq 20) \\
\text { CRP } 6 \mathrm{mg} / \mathrm{dl} & (\text { Norm }<10) \\
\text { Leukozyten } 8,2 \times 10^{3} / \mathrm{ml} & (\text { Norm 3,0-9,6) } \\
\text { Hämoglobin } 133 \mathrm{~g} / \mathrm{dl} & (\text { Norm 13,4-17,0) } \\
\text { Thrombozyten 430x103/ml } \uparrow & (\text { Norm 150-400) } \\
\text { (Transaminasen, Cholestaseparameter, } \\
\text { Nierenretentionsparameter, Elektrolyte normal) }\end{array}$ & $\begin{array}{l}\text { Die Entzündungszeichen sind wieder normalisiert, } \\
\text { die Thrombozyten minimal erhöht, wahrscheinlich } \\
\text { reaktiv bei abgelaufener Entzündung. }\end{array}$ \\
\hline
\end{tabular}

Therapie des Patienten und Verlauf

Auf Wunsch des Patienten wechseln wir in Rücksprache mit dem betreuenden Gastroenterologen auf eine Erhaltungstherapie mit Escherichia coli Nissle 1917 (Mutaflor $^{\circledR}$ ). Dabei beginnen wir mit einer tiefen Dosis (initial eine Kapsel à $0,5-5 \times 10^{9}$ Bakterien täglich) und steigern bei guter Verträglichkeit dann langsam zur Maximaldosis von zwei Kapseln à 2,5-25×109 Bakterien pro Tag.

Im weiteren Verlauf verträgt der Patient die neue Therapie gut ohne wesentliche gastrointestinale

Nebenwirkungen. Die Dauer bis zum nächsten Colitis-Schub ist mit neun Monaten vergleichbar zu den vorigen

Schüben (8, 10 bzw. 6 Monate). 


\section{Fragen zu Probiotika bei gastrointestinalen Erkrankungen}

\section{Frage 1}

Welche Aussage über Probiotika ist richtig? (Einfachauswahl, 1 richtige Antwort)

a) Probiotika kommen in Nahrungsmitteln vor.

b) Einige Probiotika können bakterizide Toxine produzieren.

c) Probiotika sind definiert als nicht-verdauliche Nahrungsbestandteile, die die Darmflora positiv beeinflussen, indem sie das Wachstum oder die Aktivität einer oder mehrerer Bakterienarten im Darm gezielt anregen.

d) VSL\# $3^{\circledR}$ ist ein Gemisch aus acht probiotischen Keimen.

e) Angesichts der heterogenen Studienlage können Probiotika bei Morbus Crohn nicht generell empfohlen werden.

\section{Frage 2}

Welche Aussagen über Saccharomyces boulardii treffen zu? (Mehrfachauswahl; mehrere richtige Antworten) Saccharomyces boulardii
a) ist ein Hefepilz.
b) kann auch bei Polymorbidität und schweren Begleiterkrankungen bedenkenlos eingesetzt werden.
c) ist effektiv in der Prophylaxe antibiotika-assoziierter Diarrhö.
d) konnte in einer Studie das Risiko eines Rezidivinfekts mit Clostridium difficile unter antibiotischer Therapie senken.
e) ist Probiotikum der Wahl beim Reizdarmsyndrom.

\section{Frage 3}

Welche Aussagen für den Einsatz von Probiotika bei chronisch-entzündlichen Darmerkrankungen sind richtig? (Mehrfachauswahl; mehrere richtige Antworten)

a) Beim akuten Schub einer Colitis ulcerosa sollen Probiotika standardmässig eingesetzt werden.

b) Escherichia coli Nissle 1917 zeigte sich in der Schubprophylaxe der Colitis ulcerosa gleich effektiv wie Mesalazin (5-Aminolsalicylsäure, 5-ASA) und kann daher alternativ eingesetzt werden.

c) VSL\#3 wirkt nur für die Therapie einer akuten Pouchitis.

d) Probiotika sind beim M. Crohn angesichts der Datenlage generell zu empfehlen.

e) Für VSL\#3 zeigte sich ein Nutzen in der Remissionserhaltung und in der Schubtherapie einer Pouchitis.

\section{Frage 4}

1. Probiotika können bei einigen gastrointestinalen Erkrankungen eingesetzt werden, weil

2. alle probiotischen Keime den gleichen, «klassenspezifischen» Effekt haben. (Einfachauswahl, 1 richtige Antwort)
a) 1. richtig, 2. falsch
b) 2. falsch, 3. richtig ((Autor: was ist «3.»?))
c) alles richtig
d) alles falsch
e) 1. richtig, 2. richtig, «weil» falsch

\section{Frage 5}

Welche Antworten zum Einsatz von Probiotika bei gastrointestinalen Erkrankungen sind richtig?

(Mehrfachauswahl, mehrere richtige Antworten) 
a) Weitere Studien sind nötig, um Empfehlungen zu Wahl und Dosierung von Probiotika abgeben zu können.

b) Probiotika können bei akutem Durchfall die Durchfalldauer reduzieren.

c) Die Wahl eines Probiotikums für eine spezielle Indikation sollte sich nach der aktuellen Studienlage richten.

d) Bei schwerer Pankreatitis sollen Probiotika eingesetzt werden.

e) Die Kosten für die regelmässige Einnahme von probiotischen Joghurts werden von allen Krankenkassen übernommen.

\section{Korrespondenzadresse}

Dr. med. Kemal Budak

Klinik und Poliklinik für Innere Medizin

UniversitätsSpital Zürich

Rämistrasse 100

8091 Zürich

kemal.budak@usz.ch

\section{Autoren}

Klinik und Poliklinik für Innere Medizin, Universitätsspital Zürich ${ }^{1}$; Klinik für Gastroenterologie und Hepatologie, Stadtspital Triemli, Zürich ${ }^{2}$

${ }^{1} \mathrm{~S}$. Braun, ${ }^{1} \mathrm{~K}$. Budak, ${ }^{2} \mathrm{~S}$. Vavricka

\section{Nützliche Literatur}

Floch MH, Walker WA, Madsen K, Sanders ME, Macfarlane GT, Flint HJ, et al.: Recommendations for probiotic use-2011 update. J Clin Gastroenterol. 2011; 45: S168-S171.

Floch MH, Walker WA, Guandalini S, Hibbers P, Gorbach S, Surawicz C, et al.: Recommendations for Probiotic use-2008. J Clin Gastroenterol. 2008; 42:S104-S108.

Meier R. Probiotika - Die Bedeutung der intestinalen Flora für die Genese und Therapie gastrointestinaler Erkrankungen. Forum Med Suisse. 2007; 7:184-191.

Sartor RB, LaMont JT, Grover S. Probiotics for gastrointestinal diseases. UpToDate, Abfrage am 08.02.2012.

\section{Bibliographie}

\section{Literaturverweise im Text:}

1. Sazawal S, Hiremath G, Dhingra U, Malik P, Deb S, Black RE: Efficacy of probiotics in prevention of acute diarrhoea: a meta-analysis of masked, randomised, placebo-controlled trials. Lancet Infect Dis 2006; 6: 374-382. 2. Hempel S, Newberry SJ, Maher AR, Wang Z, Miles JN, Shanman R, et al.: Probiotics for the Prevention and Treatment of Antibiotic-Associated Diarrhea: A Systematic Review and Meta-analysis. JAMA 2012; 307:19591969.

3. Allen SJ, Martinez EG, Gregorio GV, Dans LF: Probiotics for treating acute infectious diarrhoea. Cochrane Database Syst Rev 2010; 11: CD003048.

4. Canani RB, Cirillo P, Terrin G, Cesarano L, Spagnuolo MI, De Vicenzo A, et al.: Probiotics for treatment of acute diarrhoea in children: randomised clinical trial of five different preparations. BMJ 2007; 335:340. 
5. Guandalini S: Probiotics for children with diarrhea: an update. L Clin Gastroenterol 2008; 42: S53-S57.

6. Pillai A, Nelson R: Probiotics for treatment of Clostridium difficile-associated colitis in adults. Cochrane Database Syst Rev 2008; 1: CD004611.

7. Davidson LE, Hibberd PL, Calderwood SB, Baron EL, et al. Clostridium difficile and probiotics. UpToDate, Abfrage am 08.02.2012.

8. Butterworth AD, Thomas AG, Akobeng AK: Probiotics for induction of remission in Crohn's disease. Cochrane Database Syst Rev 2008; 3: CD006634.

9. Rolfe VE, Fortun PJ, Hawkey CJ, Bath-Hextall F: Probiotics for maintenance of remission in Crohn's disease. Cochrane Database Syst Rev 2006; 4: CD004826.

10. Kruis W, Fric P, Pokrotnieks J, Lukas M, Fixa B, Kascak M, et al.: Maintaining remission of ulcerative colitis with the probiotic Escherichia coli Nissle 1917 is as effective as with standard mesalazine. Gut 2004; 53:16171623.

11. Zocco MA, dal Verme LZ, Cremonini F, Piscaglia AC, Nista EC, Candelli M, et al.: Efficacy of lactobacillus GG in maintaining remission of ulcerative colitis. Aliment Pharmacol Ther 2006; 23:1567-1574.

12. Sood A, Midha V, Makharia GK, Ahuja V, Singal D, Goswami P, et al.: The probiotic preparation, VSL\#3 induces remission in patients with mild-to-moderately active ulcerative colitis. Clin Gastroenterol Hepatol 2009; 7: 1202-1209.

13. Bibiloni R, Fedorak RN, Tannock GW, Madsen KL, Gionchetti P, Campiere M, et al.: VSL\#3 probiotic-mixture induces remission in patients with active ulcerative colitis. Am J Gastroenterol 2005; 100: 1539-1546.

14. Tursi A, Brandimarte G, Papa A, Giglio A, Elisei W, Giorgetti GM, et al.: Treatment of relapsing mild-tomoderate ulcerative colitis with the probiotic VSL\#3 as adjunctive to a standard pharmaceutical treatment: a double-blind, randomized, placebo-controlled study. Am J Gastroenterol 2010; 105: 2218-2227.

15. Holubar SD, Cima RR, Sandborn WJ, Pardi DS. Treatment and prevention of pouchitis after ileal pouch-anal anastomosis for chronic ulcerative colitis. Cochrane Database Syst Rev 2010; 6: CD001176.

16. Fuccio L, Guido A, Eusebi LH, Laterza L, Grilli D, Cennamo V, et al.: Effects of probiotics for the prevention and treatment of radiation-induced diarrhea. J Clin Gastroenterol 2009; 43: 506-513.

17. Chmielewska A, Szajewska $\mathrm{H}$ : Systematic review of randomised controlled trials: probiotics for functional constipation. World J Gastroenterol 2010; 16: 69-75.

18. Brenner DM, Moeller MJ, Chey WD, Schoenfeld P: The utility of probiotics in the treatment of irritable bowel syndrome: a systematic review. Am J Gastroenterol 2009; 104: 1033-1049.

19. Levri KM, Ketvertis K, Deramo M, Merenstein JH, D’Amico F: Do probiotics reduce adult lactose intolerance? A systematic review. J Fam Pract 2005; 54: 613-620.

21. Szajewska H, Horvath A, Piwowarczyk A. Meta-analysis: the effects of Saccharomyces boulardii supplementation on Helicobacter pylori eradication rates and side effets during treatment. Aliment Pharmacol Ther 2010; 32: 1069-1079.

21. Besselink MG, van Santvoort HC, Buskens E, Bormeester MA, van Goor H, Timmerman HM, et al.: Probiotic prophylaxis in predicted severe acute pancreatitis: a randomised, double-blind, placebo-controlled trial. Lancet. 2008; 371: 651-659.

21. McGee RG, Bakens A, Wiley K, Riordan SM, Webster AC: Probiotics for patients with hepatic encephalopathy. Cochrane Database Syst Rev 2001; 11: CD008716.

\section{2. ((Autor: Referenz fehlt))}

23. Holte K, Krag A, Gluud LL. Systematic review and meta-analysis of randomized trials on probiotics for hepatic encephalopathy. Hepatol Res 2012 Mar 29 [Epub ahead of print] 

10.1024/1661-8157/a001042

((PX 18_Rubrik CME-Antworten))

\section{Antworten zu den Fragen zu Probiotika bei gastrointestinalen Erkrankungen aus PRAXIS Nr. 17}

Frage 1

Antwort c) ist richtig.

Diese Beschreibung trifft auf Präbiotika wie z.B. Lactulose zu.

Ad d): VSL\#3 ${ }^{\circledR}$ enthält u.a. 4 Lactobacillen (L. acidophilus, L. plantarum 299v, L. casei, L. bulgaricus), 3 Bifidobacterien (B. breve, B. longum, B. infantis) und Streptococcus thermophilus.

Ad e): In früheren Studien senkten u.a. Escherichia coli Nissle 1917, Saccharomyces boulardii und VSL\#3 ${ }^{\circledR}$ die Rezidivrate beim M. Crohn. In neueren Metaanalysen zeigt sich kein eindeutiger Nutzen.

\section{Frage 2}

Die Antworten a), c) und d) sind richtig.

Ad b): Bei schweren Komorbiditäten wurden Fungämien mit Saccharomyces boulardii beobachtet. Ein Einsatz bei schwer erkrankten, polymorbiden Patienten empfiehlt sich nicht.

Ad e): Eine klare Evidenz für den Nutzen von Probiotika beim Reizdarmsyndrom fehlt bislang. Ein möglicher Benefit besteht möglicherweise bei der Einnahme von VSL\# $3^{\circledR}$ beim Durchfall-dominierten Colon irritabile.

\section{Frage 3}

Die Antworten b) und e) sind richtig.

Ad a): In einigen Studien wurde bei mildem bis mittelschwerem Schub einer Colitis ulcerosa ein Nutzen von VSL\#3 ${ }^{\circledR}$ gefunden, sodass möglicherweise manche Probiotika auch zur Therapie eines akuten Schubs wirksam sind. Für einen standardmässigen Einsatz fehlt aber bislang klare Evidenz.

Ad b): Bei der Colitis ulcerosa zeigte sich Escherichia coli Nissle 1917 effektiv im Remissionserhalt. Ad c) und e): Die Studienlage zeigt einen Nutzen von VSL\#3 bei der Prophylaxe und Rezidivprophylaxe einer Pouchitis, nicht aber bei der akuten Therapie.

Ad d): Die derzeitigen Studien sind zu heterogen, als dass Probiotika zur Schubbehandlung oder Schubprophylaxe empfohlen werden könnten.

\section{Frage 4}

Antwort a) ist richtig.

Bei einigen Erkrankungen gibt es Evidenz für einen Benefit von Probiotika, z.B. bei der Prävention von Antibiotika-assoziiertem Durchfall, bei der Behandlung einer akuten Durchfallerkrankung, beim Remissionserhalt der Colitis ulcerosa oder bei der Prophylaxe einer Pouchitis. Generell unterscheiden sich die Probiotika in ihrer Wirkung bei speziellen Indikationen, so dass kein «klassenspezifischer» Effekt besteht und für jede Indikation das in den vorhandenen Studien effektivste Probiotikum in der entsprechenden Dosis verwendet werden sollte.

\section{Frage 5}

Die Antworten a), b) und c) sind richtig.

Ad d): Bei schwerer Pankreatitis führten Probiotika in einer Studie zu einer erhöhten Mortalität, sodass sie für diese Indikation nicht eingesetzt werden sollten. 
Ad e): In der Schweiz werden die Kosten von Probiotika-haltigen Nahrungsmitteln nicht von den Krankenkassen übernommen. Für einige wenige Probiotika, z.B. Saccharomyces boulardii und Enterococcus faecium SF68, erfolgt die Kostenübernahme bei bestimmten Indikationen. 